\title{
Estructura poblacional de Taxodium mucronatum en condiciones contrastantes de perturbación en el estado de Querétaro, México
}

\section{Population structure of Taxodium mucronatum under contrasting conditions of disturbance in the State of Querétaro, Mexico}

\author{
E. Gabriela Enríquez-Peña* y Humberto Suzán-Azpiri \\ Escuela de Biología, Universidad Autónoma de Querétaro, Avenida de las Ciencias s/n, 76230 Querétaro, Querétaro, México. \\ *Correspondencia: yaska73@yahoo.com.mx
}

\begin{abstract}
Resumen. Taxodium mucronatum es una especie riparia sujeta al efecto de perturbaciones humanas. En 8 sitios bajo condiciones contrastantes de perturbación se comparó la estructura poblacional y el crecimiento individual de la especie por 2 años. Se establecieron 2 cuadrantes de $500 \mathrm{~m}^{2}$ en cada rodal, donde se registró altura, área basal, cobertura, edad, incremento radial, producción de conos y crecimiento de brotes de cada individuo $>5 \mathrm{~cm}$ de diámetro de circunferencia del tronco a 1.50 metros. La estructura individual denotada por la altura, área basal y cobertura se vio influenciada por la condición de perturbación (MANOVA $F=6.875, p<.0001$ ), sin haber relación entre la edad y el diámetro de los árboles. Las inundaciones prolongadas alteraron el crecimiento radial, produciendo engrosamiento temporal en los troncos. La producción de conos por sitio fue significativamente diferente (año $2002 F=6.324, p<.0001$; año $2003 F=$ $10.013, p<.0001$ ); también se presentaron diferencias en el crecimiento de brotes (primera y última medición, Panales $[F=8.324, p=0.032]$ y El Trapiche $[F=8.635, p=0.030]$ ). La temperatura, la humedad disponible, las inundaciones, la materia orgánica depositada en el sustrato, los cambios hidrológicos asociados a la geomorfología y las perturbaciones causadas por el hombre afectan el crecimiento vegetativo y el desempeño reproductivo de la especie.
\end{abstract}

Palabras clave: sabino, ahuehuete, vegetación riparia, edad, crecimiento, condiciones hidrológicas, temperatura, precipitación.

\begin{abstract}
Taxodium mucronatum is a riparian species subjected to anthropocentric pressures. In 8 sites under contrasting disturbance conditions we compared the population structure and individual growth for 2 years, implementing two 500 $\mathrm{m}^{2}$ quadrats in each population where we measured for each individual $>5 \mathrm{~cm}$ the diameter at $1.5 \mathrm{~m}$, height, basal area, canopy cover, age, radial growth, cone production and shoot growth. Individual structure denoted by height, basal area and cover was influenced by the disturbance condition (MANOVA $F=6.875, p<.0001$ ), without any relationship between age and diameter of the trees. Prolonged inundations change the radial growth producing a temporal expansion of the stems. Cone production differed significantly (year $2002 F=6.324, p<.0001 ; 2003 F=10.013, p<.0001$ ), with local differences in shoot growth (first and last measurements, Panales $[F=8.324, p=0.032]$ and El Trapiche $[F=8.635$, $p=0.030]$ ). Temperature, available humidity, inundations, organic matter in the soils and human induced disturbances affect the vegetative growth and reproductive performance of the species.
\end{abstract}

Key words: Taxodium mucronatum, riparian vegetation., age, radial growth, shoot growth, hydrological conditions, temperature, precipitation

\section{Introducción}

México es uno de los países con mayor diversidad de organismos vegetales en el planeta. Los diferentes tipos de vegetación son el resultado de una compleja fisiografía, geología y variedad de climas, que en conjunto determinan un vasto mosaico de ecosistemas que desempeñan un papel muy importante en el desarrollo de las comunidades acuáticas y riparias (Lot et al., 1998; Rzedowski, 1998).

La vegetación que se desarrolla en los márgenes

Recibido: 23 abril 2008; aceptado: 10 agosto 2010 de los ríos, arroyos o manantiales se conoce como bosque de galería o vegetación riparia. Se distingue porque forma corredores naturales entre los sistemas acuático y terrestre que albergan una gran diversidad de especies y presentan un gran dinamismo ecológico. Los géneros más característicos en este tipo de vegetación en México son Platanus, Populus, Salix, Astianthus, Bambusa, Inga, Pachira, Carya, Ficus, Acer, Alnus, Fraxinus y Taxodium; conforman todos ellos un conjunto de especies dominantes, heterogéneo en cuanto a su fisonomía y estructura que comprende árboles de hoja perenne, decidua o parcialmente decidua. 
El género Taxodium pertence a la familia Taxodiaceae que reúne alrededor de 10 géneros y 16 especies; se distribuyen en regiones templadas del hemisferio norte y se distingue por los árboles muy longevos y de grandes dimensiones. En Norteamérica hay 3 especies de las cuales en México sólo se encuentra T. mucronatum Ten. (Rzedowski, 1978; Carranza, 1992; Alpert et al., 1999; Nilsson y Svedmark, 2002).

Varios son los nombres comunes con los que se conoce esta especie; destacan los de ahuehuete (náhuatl), pénhamu (tarasco), chuche (huasteco), matéoco (tarahumara), ciprés y sabino. Es un árbol con gran requerimiento de humedad, por lo que crece a orilla de ríos, manantiales, arroyos y canales, en altitudes entre 300 y $2100 \mathrm{~m}$ snm. Logra alcanzar hasta $30 \mathrm{~m}$ de altura y exhibe un tronco grueso con abultamientos irregulares, de corteza suave, color café rojizo y sabor astringente. Sus raíces son muy grandes y extendidas, y las ramas son robustas y torcidas, llegando a formar una copa amplia e irregular (Martínez, 1963; Carranza, 1992).

El ahuehuete muestra una amplia distribución en México, desde los estados de Sonora y Coahuila hasta Tabasco y Chiapas. Se le considera como el árbol nacional, y está ligado a importantes eventos históricos (Carranza, 1992). Sin embargo, es una especie amenazada, de rango limitado y sujeta al efecto de las perturbaciones provocadas por el hombre (Farjon et al., 1993). En el estado de Querétaro crece generalmente a orillas de los ríos San Juan, Estórax, Moctezuma, Jalpan y Santa María (Zamudio et al., 1992; Suzán et al., 2007).

Los beneficios que aporta la vegetación riparia al ecosistema son diversos. Según Patten (1998), evita la erosión del suelo al estabilizar los sedimentos, permite la filtración de nutrientes, reduce la cantidad de materia arrastrada corriente abajo, mejora la calidad del agua, disminuye la velocidad del flujo permitiendo su curso sinuoso, permite la recarga en el suelo y el mantenimiento de un espejo de agua elevado. Este tipo de vegetación se considera de gran importancia por su belleza y beneficio recreativo; sin embargo, ha sido objeto de frecuentes perturbaciones naturales y causadas por el hombre, como las agrícolas, pecuarias, industriales y de construcción (Malanson, 1993; Allan, 1995). Diversos estudios señalan que entre las principales causas de perturbación está el flujo del agua, que afecta el proceso de regeneración por semillas o brotes (Gecy y Wilson, 1990); por otra parte, la disponibilidad de agua, la fertilidad del suelo y la contaminación influyen en el crecimiento reproductivo (Kozlowski y Pallardy, 1997); la compactación del suelo e inundaciones prolongadas, disminuyen el crecimiento, ocasionando senescencia foliar, daño y abscisión (Kozlowski, 1985) y asimismo, la construcción de terrazas fluviales (Treviño et al., 2001).
El objetivo del presente estudio es comparar la estructura poblacional de Taxodium mucronatum, con respecto a la altura, área basal, cobertura y edad, así como el incremento radial, conos producidos y crecimiento de brotes, entre sitios que presentan condiciones contrastantes de perturbación en sistemas riparios en el estado de Querétaro, en un periodo aproximado de 2 años.

\section{Materiales y métodos}

Área de estudio. El estado de Querétaro se ubica en la República Mexicana, limitado entre los $21^{\circ} 40^{\prime}$ y $20^{\circ} 01^{\prime} \mathrm{N}$, y los $99^{\circ} 03^{\prime}$ y $100^{\circ} 36^{\prime}$ O. Está conformado por la Provincia de la Sierra Madre Oriental, la Provincia de la Mesa del Centro y la Provincia del Eje Neovolcánico, ubicadas en la Subprovincia Llanuras y Sierras de Querétaro e Hidalgo (Zamudio et al., 1992; INEGI, 2001a). Lo dividen las Regiones Hidrológicas Núm. 12 Lerma-Santiago y Núm. 26 Pánuco (INEGI, 2001a; SAGARPA, 2003), contando únicamente con 2 afluentes permanentes, el Moctezuma y el Santa María. La geología que predomina al noreste son rocas sedimentarias con suelos tipo luvisoles, rendzinas y litosoles; y hacia el suroeste formaciones volcánicas, con suelos feozem y vertisol (Zamudio et al., 1992).

El estado presenta 3 áreas climáticas bien definidas: $a)$ la zona norte con climas cálidos y semicálidos tipo $\mathrm{A}(\mathrm{w})$, $\mathrm{ACm}$ y Acw, b) la región centro con climas secos y semisecos tipo BS1(h'), BS1h, BS1k y BSh, y c) la porción sur con climas templados subhúmedos tipo $\mathrm{C}(\mathrm{w})$. La precipitación anual oscila de 630 a $860 \mathrm{~mm}$ (INEGI, 2004a).

La variabilidad fisiográfica, geológica y climática en la entidad permite una gran diversidad de la vegetación terrestre; sin embargo, la vegetación acuática y subacuática alcanza un desarrollo relativamente escaso. Los bosques de galería se desarrollan a orillas de los ríos San Juan, Estórax, Moctezuma, Jalpan y Santa María, con especies arbóreas como Fraxinus uhdei, Ficus cotinifolia, Populus fremontii, Salix chilensis y T. mucronatum. Esta vegetación se ha visto afectada por la agricultura, ganadería, asentamientos, industrias, vías de comunicación y contaminación (Zamudio et al., 1992; INEGI, 2004b). Se ha registrado T. mucronatum en los municipios de Arroyo Seco, Jalpan, Landa, Pinal de Amoles, San Joaquín, Querétaro, Tolimán, Cadereyta, Tequisquiapan, San Juan del Río y Amealco.

Diseño experimental. Se seleccionaron 8 sitios en 5 municipios del estado de Querétaro que presentaban sabinos y condiciones contrastantes de perturbación y cauces de agua permanentes o semipermanentes (Fig.1). Los sitios de muestreo se agruparon de acuerdo a 3 condiciones contrastantes de perturbación: 1) bien conservado, 2) 
ligeramente perturbado y 3) muy perturbado (Cuadro 1). Esta clasificación se obtuvo a partir de datos obtenidos de la Comisión Nacional del Agua (2001), sobre el Índice de Calidad de Agua de acuerdo con su uso, criterios de perturbación (Olvera et al. 1996), de datos geológicos del Instituto Nacional de Geografía e Informática (INEGI, 2001b; 2001c; 2002a; 2002b; 2003a; 2003b) y de observaciones a las actividades agrícolas y frecuencia de paseantes hechas en campo.

Con base en la accesibilidad al arbolado y al tipo de perturbación, en cada rodal se establecieron al azar 2 cuadrantes permanentes de $500 \mathrm{~m}^{2}(10 \times 50)$, distribuidos a lo largo del cauce del río o arroyo (Tardif y Bergeron, 1999). Para comparar el efecto del embalse, se establecieron 2 cuadrantes en los sitios Presa de Galindo 1 (antes de la presa) y en Presa de Galindo 2 (después de la presa), mientras que en El Trapiche y Puente de Ayutla sólo se estableció 1 , debido a que el tipo de perturbación fue muy semejante.

Se registraron las variables altura, área basal, cobertura, edad, incremento radial, conos y semillas producidos, y crecimiento de brotes en todos los individuos por cuadrante (Suzán et al. 2007). Se midió la circunferencia del tronco $(>5 \mathrm{~cm})$ con cinta métrica, a una altura de $1.50 \mathrm{~m}$ desde el suelo, evitando contrafuertes (Tardif y Bergeron, 1999). La altura se obtuvo con un clinómetro marca Suunto y el área basal se calculó aplicando la fórmula: $A B=C^{2}$ / 4 (3.1416) donde $\mathrm{C}=$ circunferencia. La cobertura de la copa se obtuvo al promediar la longitud total de 2 radios, y mediante la fórmula del área: $\pi \mathrm{r}^{2}$, donde $\pi=3.1416 \mathrm{y} \mathrm{r}=$ radio. El incremento radial se calculó sólo en 6 rodales con un microdendrómetro que se sujetó al tronco por medio de tornillos fijos durante el periodo comprendido de mayo de 2000 a noviembre de 2003 (45 meses). Se monitoreó el incremento en forma mensual en Panales, Presa de Galindo 2 y El Charcón, mientras que en Barranca de Amealco/SJR (San Juan del Río), El Trapiche y Tequisquiapan, el tiempo de muestreo fue variable (entre 8 y 23 meses). En Puente de Ayutla no se midió el incremento por la semejanza en su condición de perturbación con El Trapiche. Debido a las diferencias en cuanto al tiempo de medición y a la falta de registros, esta variable se analizó como el promedio del incremento radial mensual.

La producción de conos y semillas se estimó por medio del conteo de conos en una rama principal (muestreo aleatorio simple), cuantificándose luego el número total de ramas principales; asimismo, se contó el número de semillas por cono.

El crecimiento de brotes por rama se determinó por la selección aleatoria de 3 árboles, con una conformación de fuste y copa semejantes en cada sitio. Se escogieron 4 ramas con diferente orientación y fácil acceso, luego se contó en su porción terminal ( $1 \mathrm{~m}$ aprox.) el número de brotes y su longitud, clasificándose en 5 categorías: 1) 20-30 cm, 2) $31-50 \mathrm{~cm}, 3) 51-70 \mathrm{~cm}, 4) 71-100 \mathrm{~cm}$ y 5) $>100 \mathrm{~cm}$. La medición se hizo tomando la rama principal como eje central, marcándola para su identificación y considerando como brotes las ramas desarrolladas en posición perpendicular. Asimismo se determinaron diámetro, altura, cobertura y número de ramas por metro cúbico. La evaluación de brotes se efectuó de finales de febrero de 2004 (primera medición) a finales de agosto del mismo año (última medición).

La edad promedio en árboles adultos se calculó mediante núcleos de crecimiento obtenidos a $1.30 \mathrm{~m}$ desde la base del suelo con un taladro Pressler, marca Haglöf de $500 \mathrm{~mm}$ (20") de 2 filos (Trenard, 1982). El tamaño de muestra fue de 15 a 20 árboles con 2 núcleos por árbol (Fritts, 1976). En Presa de Galindo 1, sólo se muestrearon 7 árboles, ya que se consideró como la continuación del arbolado proveniente de Barranca Amealco/SJR. En El Trapiche no se obtuvieron núcleos de crecimiento, porque se contó con datos aledaños a la zona, analizados en 1999.

Los núcleos se analizaron en el Laboratorio de Dendrocronología del Instituto Nacional de Investigaciones Forestales, Agrícolas y Pecuarias, Centro Nacional de Investigación Disciplinaria en Relación Agua-Suelo Planta-Atmósfera (INIFAP CENID-RASPA) ubicado en Gómez Palacio, Durango. Se montaron sobre bases de madera para someterlos a un proceso de pulido (lija .gruesa a fina) y se contaron con un microscopio estereoscópico marca Motic, resolución 10 a 20×. La edad en plántulas se analizó en 73 individuos que se clasificaron en 4 categorías de altura: 1) $50 \mathrm{~cm}(50 \mathrm{~cm} \mathrm{a}<90 \mathrm{~cm})$, 2) $100 \mathrm{~cm}(\geq 90 \mathrm{~cm}$ a $<140 \mathrm{~cm}), 3) 150 \mathrm{~cm}(\geq 140 \mathrm{~cm}<190 \mathrm{~cm})$, 4) $200 \mathrm{~cm}$ $(\geq 190 \mathrm{~cm} \mathrm{a}<310 \mathrm{~cm}$ ). Se cortaron en su base obteniendo pequeñas rodajas, que fueron pulidas (lijas Núm. 80,120 y 220) para resaltar los anillos de crecimiento. Se analizaron los datos de la categoría de $150 \mathrm{~cm}$ para evaluar el tiempo necesario en alcanzar el diámetro a la altura del pecho. Este procedimiento sólo se hizo en Panales, Barranca de Amealco/SJR, Presa de Galindo, Trapiche y El Charcón.

En la Comisión Nacional del Agua (Gerencia Estatal en Querétaro), se revisaron los registros de la precipitación y temperatura promedio anuales durante el periodo entre 1984-2004 de las estaciones meteorológicas más cercanas a los sitios de estudio. Las estaciones fueron: Tolimán, Mpio. Tolimán; Galindo, Mpio. San Juan del Río; Ayutla, Mpio. Arroyo Seco; Paso de Tablas, Mpio. Tequisquiapan; y El Salitre, Mpio. Cadereyta

Análisis estadístico

Estructura poblacional. Se aplicó un análisis de varianza (ANOVA) y un análisis multivariado de la varianza (MANOVA) de la altura, el área basal y la cobertura, con 
Cuadro 1. Ubicación geográfica y grado de perturbación de los sitios de estudio en el estado de Querétaro

\begin{tabular}{|c|c|c|c|c|c|c|}
\hline Sitio & Municipio & Ubicación & $\begin{array}{l}\text { Altitud } \\
\text { (msnm) }\end{array}$ & Perturbación & Condición & Suelo \\
\hline Panales & Tolimán & $\begin{array}{c}20^{\circ} 52^{\prime} 17^{\prime} \\
99^{\circ} 59^{\prime} 19.2 ”\end{array}$ & 1672 & $\begin{array}{l}\text { Pastoreo ligero. } \\
\text { Cauce de agua } \\
\text { permanente. Control del } \\
\text { paso vehicular y humano. } \\
\text { Ausencia de erosión de } \\
\text { suelo. }\end{array}$ & $\begin{array}{c}\text { Bien } \\
\text { conservado }\end{array}$ & $\begin{array}{c}\text { Roca } \\
\text { sedimentaria } \\
\text { (conglomerado) }\end{array}$ \\
\hline $\begin{array}{l}\text { Barranca de } \\
\text { Amealco / San } \\
\text { Juan del Río }\end{array}$ & $\begin{array}{l}\text { San Juan del } \\
\text { Río (límites } \\
\text { con Amealco) }\end{array}$ & $\begin{array}{c}20^{\circ} 20^{\prime} 24.8^{\prime \prime} \\
100^{\circ} 07^{\prime} 21.5^{\prime \prime}\end{array}$ & 2060 & $\begin{array}{l}\text { Pastoreo ligero. } \\
\text { Cauce de agua } \\
\text { permanente. Visitas de } \\
\text { paseantes. Ausencia de } \\
\text { erosión de suelo. }\end{array}$ & $\begin{array}{c}\text { Bien } \\
\text { conservado }\end{array}$ & $\begin{array}{c}\text { Roca ígnea } \\
\text { extrusiva (toba } \\
\text { ácida) }\end{array}$ \\
\hline $\begin{array}{l}\text { Presa de } \\
\text { Galindo } 1 \\
\text { (antes de la } \\
\text { presa) }\end{array}$ & $\begin{array}{l}\text { San Juan del } \\
\text { Río }\end{array}$ & $\begin{array}{c}20^{\circ} 22^{\prime} 43.8^{\prime \prime} \\
100^{\circ} 05,56.6 "\end{array}$ & 1970 & $\begin{array}{l}\text { Pastoreo moderado. Cauce } \\
\text { controlado por la presa de } \\
\text { la localidad. Erosión de } \\
\text { suelo leve. }\end{array}$ & $\begin{array}{l}\text { Ligeramente } \\
\text { perturbado }\end{array}$ & Roca volcánica \\
\hline $\begin{array}{c}\text { Presa de } \\
\text { Galindo } 2 \\
\text { (después de la } \\
\text { presa) }\end{array}$ & $\begin{array}{l}\text { San Juan del } \\
\text { Río }\end{array}$ & $\begin{array}{l}20^{\circ} 22^{\prime} 12.5^{\prime} \\
100^{\circ} 06^{\prime} 31.3 "\end{array}$ & 2000 & $\begin{array}{l}\text { Pastoreo moderado. Cauce } \\
\text { controlado por la presa de } \\
\text { la localidad. Erosión de } \\
\text { suelo leve. }\end{array}$ & $\begin{array}{l}\text { Ligeramente } \\
\text { perturbado }\end{array}$ & Roca volcánica \\
\hline El Trapiche & Arroyo Seco & $\begin{array}{l}21^{\circ} 19.67^{\prime} 00^{\prime \prime} \\
99^{\circ} 31.27^{\prime} 00^{\prime \prime}\end{array}$ & 720 & $\begin{array}{l}\text { Pastoreo moderado. } \\
\text { Cauce de agua } \\
\text { permanente. Alta } \\
\text { pedregosidad en suelo. } \\
\text { Erosión de suelo } \\
\text { moderada. }\end{array}$ & $\begin{array}{l}\text { Ligeramente } \\
\text { perturbado }\end{array}$ & $\begin{array}{c}\text { Roca } \\
\text { sedimentaria } \\
(\text { caliza })\end{array}$ \\
\hline $\begin{array}{l}\text { Puente de } \\
\text { Ayutla }\end{array}$ & Arroyo Seco & $\begin{array}{c}21^{\circ} 23.7^{\prime} 00^{\prime \prime} \\
99^{\circ} 35^{\prime} 00^{\prime \prime}\end{array}$ & 650 & $\begin{array}{l}\text { Pastoreo moderado. } \\
\text { Cauce de agua } \\
\text { permanente. Extracción } \\
\text { de arena por pobladores. } \\
\text { Erosión de suelo } \\
\text { moderada. }\end{array}$ & $\begin{array}{l}\text { Ligeramente } \\
\text { perturbado }\end{array}$ & $\begin{array}{l}\text { Roca ígnea } \\
\text { extrusiva } \\
\text { (basalto) y } \\
\text { sedimentaria } \\
\text { (caliza) }\end{array}$ \\
\hline Tequisquiapan & Tequisquiapan & $\begin{array}{l}20^{\circ} 32^{\prime} 20.0 " \\
99^{\circ} 53 ’ 00.1 "\end{array}$ & 1909 & $\begin{array}{l}\text { Ausencia de pastoreo. } \\
\text { Cauce permanente } \\
\text { con poco movimiento. } \\
\text { Descarga de aguas } \\
\text { residuales. Incendios } \\
\text { fuertes. Erosión de suelo } \\
\text { fuerte. }\end{array}$ & $\begin{array}{c}\text { Muy } \\
\text { perturbado }\end{array}$ & $\begin{array}{c}\text { Rocas } \\
\text { sedimentaria } \\
\text { (arenisca y } \\
\text { conglomerado) }\end{array}$ \\
\hline El Charcón & $\begin{array}{c}\text { Cadereyta de } \\
\text { Montes }\end{array}$ & $\begin{array}{c}20^{\circ} 35^{\prime} 54.3 ” \\
99^{\circ} 44^{\prime} 4.8^{\prime \prime}\end{array}$ & 1727 & $\begin{array}{l}\text { Pastoreo intenso. } \\
\text { Cauce estático o con poco } \\
\text { movimiento en algunas } \\
\text { partes. Descarga de } \\
\text { aguas residuales. Podas } \\
\text { constantes al arbolado. } \\
\text { Suelo compacto y erosión } \\
\text { fuerte. Basurero en } \\
\text { cercanías. }\end{array}$ & $\begin{array}{c}\text { Muy } \\
\text { perturbado }\end{array}$ & $\begin{array}{l}\text { Roca ígnea } \\
\text { extrusiva (toba } \\
\text { ácida) }\end{array}$ \\
\hline
\end{tabular}


el paquete estadístico JMP (SAS, 1995), para comparar las medias de cada sitio en una sola variable y considerar simultáneamente todas las variables. Se aplicó un análisis de regresión $\left(r^{2}\right)$ entre la edad estimada y las variables diámetro y altura; para definir si existe relación o no entre el tamaño de la especie y la longevidad que presenta.

Producción de conos y semillas. Por medio del programa JMP (SAS, 1995) se aplicó un ANOVA para comparar la producción de conos y de semillas durante los años 2002 y 2003. También se ejecutó un análisis de correlación (coeficiente $r$ ) para conocer la medida de la asociación lineal entre el número de ramas principales producidas por individuo y el número de conos.

Crecimiento de brotes. Se utilizó la prueba de $F$ (probabilidad de una sola cola de que las varianzas de 2 matrices no sean significativamente diferentes) para comparar el número de brotes encontrados en la primera y última medición (Programa Excel de Microsoft, Office 2000); y se aplicó el coeficiente $r$ para determinar la asociación lineal entre las variables diámetro, altura y cobertura de los árboles bajo estudio de brotes, y la producción de ramas por metro cúbico (SAS, 1995).

\section{Resultados}

El presente trabajo consistió en comparar el comportamiento de las variables de estudio entre los sitios, es decir, entender el papel que ejerce el grado de perturbación en el desarrollo de la especie T. mucronatum.

Estructura poblacional. Los resultados del ANOVA y MANOVA indicaron una diferencia significativa entre las variables altura, área basal y cobertura. Los sitios Panales, El Trapiche y Tequisquiapan presentaron los valores promedio más altos en las 3 variables, mientras que en Presa de Galindo las diferencias sólo se apreciaron en área basal y cobertura, siendo menores en el rodal antes de la presa. Barranca de Amealco/SJR, El Charcón y Puente de Ayutla exhibieron una cobertura y área basal promedio bajas (Cuadro 2). La edad promedio en plántulas fue de $9.9 \pm$ 0.99 años ( $\mathrm{n}=19)$, lo cual advierte que un sabino alcanza una edad aproximada de 10 años a la altura de $150 \mathrm{~cm}$; por tanto, se sumaron 10 años a la edad calculada en los árboles adultos. Respecto a los adultos, se encontró que los sitios Barranca de Amealco/SJR y Presa de Galindo 1 son los rodales más viejos, seguidos de Panales y El Trapiche, mientras que los rodales más jóvenes se encontraron en El Charcón y Tequisquiapan (Cuadro 3).

El análisis de regresión no arrojó relación alguna entre la edad y el diámetro. El rodal Puente de Ayutla presentó el valor de regresión más elevado en comparación con los demás sitios $\left(r^{2}=0.412\right)$, el cual se consideró poco significativo para definir que el aumento en diámetro es directamente proporcional a la edad. Asimismo, no hubo relación alguna entre la edad y la altura; sólo Puente de Ayutla mostró una $r^{2}=0.483$, que también se consideró poco significativa.

Incremento radial. Los rodales de Panales y Presa de Galindo 2 exhibieron durante los 45 meses de muestreo un incremento total promedio semejante, mientras que El Charcón mostró un incremento mayor (Cuadro 3). Los datos calculados para Barranca de Amealco/SJR y El Trapiche se obtuvieron durante periodos de tiempo diferentes, haciendo complicada su comparación. Tequisquiapan se incluyó tardíamente en el muestreo; por tanto, someterlo al análisis sería una conjetura prematura. Sin embargo, destaca el hecho de que junto con Presa de Galindo 2 y El Charcón, advirtieron en promedio un incremento radial mensual mayor, en comparación con los demás sitios (Fig. 2). De acuerdo con los datos de las estaciones meteorológicas Tolimán y El Salitre, los sitios Panales y El Charcón presentaron un incremento radial total mayor, bajo condiciones de temperatura relativamente semejantes (17 a $20{ }^{\circ} \mathrm{C}$ ) en los últimos 20 años. Presa de Galindo 2 tuvo un comportamiento similar en este mismo periodo de tiempo, pero la ausencia de datos de temperatura y precipitación durante 1988 a 1997, no permitió una comparación satisfactoria (Fig. 3).

Producción de conos y ramas principales. La producción de conos fue significativamente diferente para los años 2002 y 2003. En el 2002, Panales, Presa de Galindo 2, El Trapiche y Puente de Ayutla obtuvieron en promedio el mayor número de conos producidos por individuo, mientras que para el 2003 fueron Panales, El Trapiche y Tequisquiapan. Los rodales de Presa de Galindo 1 produjeron un número bajo de conos durante los 2 años de muestreo. En el 2003 Puente de Ayutla no se muestreó debido a problemas de logística. En el caso de Tequisquiapan, la ausencia de datos en el 2002 se debió a que se incorporó al estudio un año después (Fig. 4). En cuanto a ramas principales producidas por individuo, los sitios Panales y El Trapiche se destacaron por un valor promedio alto de $13.82 \pm 1.95$ y $11.67 \pm 0.56$, respectivamente, seguidos de Puente de Ayutla con $8.78 \pm 1.05$, Presa de Galindo 2 con $7.80 \pm 0.58$ y Tequisquiapan con $7.63 \pm 1.26$, mientras que El Charcón expuso el valor más bajo con $5.60 \pm 0.76$ ramas.

El Análisis de Correlación entre la producción de ramas y conos por individuo, indicó un Coeficiente $r$ elevado en El Charcón durante los 2 años, mientras que Tequisquiapan lo fue para el 2003. Los sitios Panales y El Trapiche revelaron en promedio un número elevado de ramas principales, sin embargo, no se encontró una asociación lineal significativa con la producción de conos. La asociación 
más baja la presentó Barranca de Amealco/SJR en el año 2002 y Presa de Galindo 2 en el 2003 (Cuadro 4).

Producción de semillas. Fue significativamente diferente en los años $2002(F=15.754, p=<.0001)$ y $2003(F=$ 21.893, $p=<.0001)$. El rodal Presa de Galindo 1 exhibió en promedio el mayor número de semillas producidas por cono en el año $2002(37.08 \pm 1.23)$, y el sitio Panales lo fue en el 2003 (37.86 \pm 1.28$)$. Barranca de Amealco/SJR obtuvo los valores más bajos durante los 2 años (2002= $23.80 \pm 2.04,2003=21.73 \pm 1.13$ ). Durante el 2002 no se hallaron conos maduros en Presa de Galindo 2, Tequisquiapan, El Trapiche y Puente de Ayutla, dificultando un análisis comparativo formal; sin embargo, se advirtió que la producción de semillas difirió entre un año y otro.

Crecimiento de brotes. En la primera medición, Panales presentó una mayor proporción de brotes en la categoría de longitud número 4 (71 a $100 \mathrm{~cm})$, mientras que Barranca de Amealco/SJR lo fue en la categoría $3(51$ a $70 \mathrm{~cm})$. Los brotes de Tequisquiapan se clasificaron en su mayoría en la categoría 2 (31 a $50 \mathrm{~cm})$ y los de El Charcón se agruparon en la 1 (20 a $30 \mathrm{~cm})$. Hubo diferencias entre los rodales Presa de Galindo 1 y Presa de Galindo 2, ya que el primero mostró un número mayor de brotes en la categoría 3 , mientras que el segundo lo fue en la categoría 1. El Trapiche exhibió un porcentaje elevado de brotes en la categoría 3. En Puente de Ayutla sólo hubo brotes en la categoría número $5(>100 \mathrm{~cm})$ (Fig. 5). En la última medición, todos los sitios evidenciaron un extenso número de brotes de 20 a $30 \mathrm{~cm}$ de longitud, siendo notorio el valor por encima del 60\% en Puente de Ayutla y El Charcón. Por el contrario, Presa de Galindo 1 mostró el porcentaje más alto de brotes en la categoría 2. A diferencia de la primera medición, la mayoría de los sitios presentaron brotes con una longitud $>100 \mathrm{~cm}$, exceptuando Puente de Ayutla y El Charcón (Fig. 5). La prueba de $F$ sólo arrojó diferencias significativas en Panales $(F=8.324, p=0.032)$ y El Trapiche $(F=8.635, p=0.030)$ en la primera y última medición.

El ANOVA exhibió también diferencias $(p \leq 0.05)$ entre el diámetro, altura y producción de ramas por metro cúbico en la primera y última medición. En cuanto al diámetro y altura promedios, El Trapiche presentó los datos más elevados. En promedio, Barranca de Amealco/SJR y El Trapiche denotaron más ramas por metro cúbico en ambas mediciones (Cuadro 5). De acuerdo con el coeficiente $r$, el diámetro y la altura no muestran una asociación lineal alta con la producción de ramas. Sólo los valores arrojados para la cobertura indican un valor significativo con el número de ramas encontradas por metro cúbico (Cuadro 6).

\section{Discusión}

Varios estudios han evaluado el efecto de las perturbaciones sobre el crecimiento de especies riparias. Se han registrado 2 tipos de causas, las directas como la agricultura y la ganadería; y las indirectas como los canales, diques o presas (Malanson, 1993); por otro lado, en la mayoría de los casos, estas alteraciones junto con la geomorfología, la luz y la temperatura provocan cambios en la estructura y dinámica de la vegetación (Naiman y Décamps, 1997); asimismo, las inundaciones prolongadas retardan el crecimiento, debido a que disminuyen el oxígeno en el suelo, o reducen el crecimiento de tallos y raíces, ya que aumentan las auxinas, el etileno y el ácido abscísico en los tallos, mientras que los niveles de giberelinas y citoquininas disminuyen; aunque especies tolerantes, como Nyssa aquatica y Taxodium distichum pueden llegar a incrementar su desarrollo debido al movimiento del cauce, y en ocasiones es evidente un "hinchamiento" de los tallos, denotando un aumento en diámetro (Kozlowski, 1985); en algunos casos los desechos orgánicos en el agua producen cambios en diámetro y área basal (biomasa en general) en Taxodium distichum y T. distichum var. nutans (Schlesinger, 1978; Brown, 1981; Hesse et al., 1998), o bien, que la presencia de un suelo fértil puede revertir el efecto negativo que provocan los bajos niveles de oxígeno, altas concentraciones de bióxido de carbono y otros gases como metano debido al agua anegada o estática (Harms, 1973). Bajo este enfoque, se puede argumentar que las diferentes condiciones de perturbación en los sitios Panales (bien conservado), El Trapiche (ligeramente perturbado) y Tequisquiapan (muy perturbado), han afectado su estructura poblacional ya que la densidad fue muy variable (Cuadro 2), descartando en primera instancia el efecto de la competencia por espacio y luz; sin embargo, las condiciones del cauce durante la temporada de lluvias y/o estiaje parece que influyen de manera preponderante en el crecimiento de T. mucronatum, ya que a pesar de las diferencias en cuanto al tipo de perturbación, mostraron una altura, área basal y cobertura mayor que los demás sitios (Cuadro 2). Las podas constantes para actividades agrícolas merman el crecimiento en cuanto a la cobertura de copa, producción de ramas principales y conos; ejemplo claro lo fue El Charcón (muy perturbado), que exhibió la cobertura promedio más baja (Cuadro 2), indicando que este tipo de perturbación afecta directamente la estructura del arbolado. El incremento radial mensual promedio fue mayor en los sitios Presa de Galindo 2 (ligeramente perturbado), Tequisquiapan y El Charcón (Fig. 2), situación que puede atribuirse a las condiciones del cauce. El desfogue del embalse a finales de la temporada de lluvias induce a 
Cuadro 2. Análisis de Varianza (ANOVA) y Análisis Multivariado de la Varianza (MANOVA) del promedio de la altura, área basal y cobertura de Taxodium mucronatum en el estado de Querétaro

\begin{tabular}{|c|c|c|c|c|}
\hline Sitio & $\begin{array}{l}\text { Altura (m) } \\
\pm \text { Error Std. }\end{array}$ & $\begin{array}{l}\text { Area Basal }\left(\mathrm{m}^{2}\right) \\
\quad \pm \text { Error Std. }\end{array}$ & $\begin{array}{c}\text { Cobertura }\left(\mathrm{m}^{2}\right) \\
\pm \text { Error Std. }\end{array}$ & $\begin{array}{l}\text { Densidad } \\
\text { (Ind. / Ha.) }\end{array}$ \\
\hline Panales & $19.85 \pm 1.01$ & $1.79 \pm 0.21$ & $203.88 \pm 30.76$ & 110 \\
\hline B. Amealco / SJR & $10.93 \pm 0.37$ & $0.64 \pm 0.13$ & $84.82 \pm 8.77$ & 150 \\
\hline Presa de Galindo 1 & $7.58 \pm 0.60$ & $0.39 \pm 0.10$ & $61.89 \pm 12.34$ & 360 \\
\hline Presa de Galindo 2 & $8.00 \pm 1.76$ & $0.50 \pm 0.11$ & $72.25 \pm 23.07$ & 50 \\
\hline El Trapiche & $14.00 \pm 0.58$ & $2.45 \pm 0.70$ & $113.39 \pm 19.35$ & 60 \\
\hline Puente de Ayutla & $9.33 \pm 1.10$ & $0.76 \pm 0.33$ & $59.54 \pm 18.06$ & 90 \\
\hline Tequisquiapan & $12.42 \pm 0.46$ & $3.40 \pm 1.26$ & $154.9 \pm 30.17$ & 190 \\
\hline \multirow[t]{3}{*}{ El Charcón } & $11.64 \pm 0.95$ & $0.54 \pm 0.19$ & $58.91 \pm 19.71$ & 200 \\
\hline & $F=20.063$ & $F=3.945$ & $F=5.567$ & \\
\hline & $p=<.0001^{*}$ & $p=<.0007^{*}$ & $p=<.0001 *$ & \\
\hline MANOVA & Wilk's Lambda $F=6.875$ & $p=<.0001^{*}$ & & \\
\hline
\end{tabular}

Cuadro 3. Valores promedio de edad e incremento radial total $(\mathrm{mm})$ de Taxodium mucronatum en el estado de Querétaro

\begin{tabular}{lcccccc}
\hline \multicolumn{1}{c}{ Sitio } & $\begin{array}{c}{ }^{c} \text { Edad estimada } \\
\pm \text { Error Std. }\end{array}$ & $\begin{array}{c}\text { Edad } \\
\text { Min. }\end{array}$ & $\begin{array}{c}\text { Edad } \\
\text { Máx. }\end{array}$ & $n$ & $\begin{array}{c}\text { Incremento } \\
\text { total } \\
\pm \text { Error Std }\end{array}$ & $\begin{array}{c}\text { Tiempo } \\
\text { (meses) }\end{array}$ \\
\hline Panales & $184.79 \pm 4.02$ & 154 & 217 & 19 & $16.74 \pm 3.94$ & 45 \\
B. Amealco / SJR & $389.07 \pm 55.15$ & 136 & 953 & 17 & $12.40 \pm 4.81$ & 23 \\
Presa de Galindo ${ }^{a}$ & $269.57 \pm 17.59$ & 200 & 334 & 7 & $15.03 \pm 3.64$ & 45 \\
El Trapiche & $188.67 \pm 19.92$ & 64 & 249 & 9 & $14.49 \pm 6.34$ & 18 \\
Puente de Ayutla ${ }^{b}$ & $120.06 \pm 20.44$ & 20 & 314 & 16 & -- & - \\
Tequisquiapan & $103.3 \pm 9.63$ & 53 & 197 & 20 & $9.84 \pm 4.69$ & 8 \\
El Charcón & $97.30 \pm 4.03$ & 48 & 130 & 20 & $36.55 \pm 6.51$ & 45 \\
\hline
\end{tabular}

a La edad se obtuvo en el rodal ubicado antes de la presa, y el incremento radial en el que se ubica después de la presa.

${ }^{\mathrm{b}}$ Puente de Ayutla no se consideró en el muestreo de incremento radial.

${ }^{c}$ Edad estimada +10 años de brinzales. 
Cuadro 4. Análisis de Correlación (Coeficiente $r$ ) entre la producción de ramas y producción conos de Taxodium mucronatum en el Estado de Querétaro

\begin{tabular}{|c|c|c|c|c|}
\hline \multirow[t]{2}{*}{ Sitio } & \multicolumn{2}{|c|}{ Año 2002} & \multicolumn{2}{|c|}{ Año 2003} \\
\hline & Coeficiente $r$ & $p$ & Coeficiente $r$ & $p$ \\
\hline Panales & 0.338 & 0.308 & 0.554 & 0.077 \\
\hline $\begin{array}{l}\text { Barranca Amealco / } \\
\text { SJR }\end{array}$ & 0.118 & 0.675 & 0.488 & 0.090 \\
\hline Presa de Galindo1 & 0.693 & 0.0002 & 0.645 & 0.0002 \\
\hline Presa de Galindo 2 & 0.780 & 0.119 & 0.058 & 0.926 \\
\hline El Trapiche & 0.199 & 0.705 & 0.304 & 0.557 \\
\hline Puente de Ayutla & 0.499 & 0.171 & --- & --- \\
\hline Tequisquiapan & --- & --- & 0.895 & $0.001 *$ \\
\hline El Charcón & 0.868 & $0.001 *$ & 0.828 & $0.003 *$ \\
\hline
\end{tabular}

$* p \leq 0.01$

Cuadro 5. Análisis de Varianza (ANOVA) del promedio de diámetro, altura, cobertura y ramas producidas por metro cúbico de Taxodium mucronatum, en el estado de Querétaro

\begin{tabular}{|c|c|c|c|c|c|}
\hline Sitio & Diámetro $(\mathrm{cm})$ & Altura (m) & Cobertura $\left(m^{2}\right)$ & $R 1$ & $R 2$ \\
\hline Panales & $72.04 \pm 19.05$ & $20.67 \pm 0.67$ & $220.43 \pm 97.68$ & 5.6 & 3.3 \\
\hline B. Amealco / SJR & $147.30 \pm 61.67$ & $17.67 \pm 2.19$ & $317.42 \pm 244.02$ & 15.3 & 16.0 \\
\hline Presa de Galindo 1 & $21.39 \pm 6.70$ & $13.67 \pm 0.88$ & $127.23 \pm 17.22$ & 3.3 & 5.6 \\
\hline Presa de Galindo 2 & $90.08 \pm 7.53$ & $15.67 \pm 2.85$ & $125.20 \pm 25.88$ & 2.3 & 3.3 \\
\hline El Trapiche & $257.51 \pm 22.54$ & $23.33 \pm 0.88$ & $459.19 \pm 76.25$ & 7.6 & 9.7 \\
\hline Puente de Ayutla & $39.47 \pm 6.77$ & $9.17 \pm 0.44$ & $151.92 \pm 37.39$ & 6.3 & 5.0 \\
\hline Tequisquiapan & $134.86 \pm 47.64$ & $16.00 \pm 3.61$ & $174.36 \pm 40.36$ & 2.3 & 2.0 \\
\hline \multirow[t]{3}{*}{ El Charcón } & $81.38 \pm 28.38$ & $14.67 \pm 1.76$ & $96.60 \pm 18.06$ & 2.3 & 3.3 \\
\hline & $F=5.656$ & $F=4.812$ & $F=1.518$ & $F=4.895$ & $F=15.940$ \\
\hline & $p=0.002 *$ & $p=0.004^{*}$ & $p=0.230$ & $p=0.004^{*}$ & $p=<.0001^{*}$ \\
\hline
\end{tabular}

R1 = Ramas por metro cúbico, primera medición.

$\mathrm{R} 2=$ Ramas por metro cúbico, última medición. 
Cuadro 6. Análisis de Correlación (Coeficiente $r$ ) entre la estructura del arbolado y número de ramas producidas por metro cúbico de Taxodium mucronatum, en el estado de Querétaro

\begin{tabular}{lccc}
\hline \multicolumn{1}{c}{ Variables } & Coeficienter & $F$ & $p$ \\
\hline Diámetro / R1 & 0.138 & 0.426 & 0.52 \\
Diámetro / R2 & 0.366 & 3.406 & 0.078 \\
Altura / R1 & 0.071 & 0.112 & 0.740 \\
Altura / R2 & 0.259 & 1.584 & 0.221 \\
Cobertura / R1 & 0.599 & 12.282 & $0.002^{*}$ \\
Cobertura / R2 & 0.433 & 5.090 & $0.034^{*}$ \\
\hline
\end{tabular}

R1 = Ramas por metro cúbico, primera medición.

R2= Ramas por metro cúbico, última medición.

periodos prolongados de inundación, poco movimento y saturación del suelo en Presa de Galindo 2, mientras que la corriente en Tequisquiapan y El Charcón, es relativamente estática o sin movimiento en algunas partes, ocasionando que el arbolado se desarrolle en condiciones anegadas.

La disponibilidad de humedad puede ser un factor determinante en el crecimiento de brotes. En general, los rodales bien conservados presentaron un mayor número de brotes con una longitud de 51 a $70 \mathrm{~cm}$ y 71 a $100 \mathrm{~cm}$ en la primera medición, denotando un crecimiento hacia las categorías de 71 a $100 \mathrm{~cm}$ y $>100$ centímetros. Presa de Galindo 2 mostró un mayor número de brotes de menor longitud en comparación con el rodal ubicado antes del embalse (Fig. 5). Fue notable la diferencia de producción de brotes $>100 \mathrm{~cm}$ entre El Trapiche y Puente de Ayutla (ligeramente perturbado) durante las 2 temporadas de medición, condición que puede atribuirse a la cantidad de materia orgánica depositada en el sustrato superficial, ya que permite una mayor retención de humedad. Sólo hubo diferencias significativas en Panales y El Trapiche en la primera y última temporada de medición de brotes; por tanto, es probable que la condición de perturbación no sólo influye directamente en el crecimiento de brotes, sino la acción conjunta de la disponibilidad del agua, las condiciones del dosel (competencia por luz y espacio) y el sustrato, determinan también este crecimiento.

Un factor relevante que se logró distinguir es la descarga continua de aguas residuales provenientes de localidades cercanas y fábricas en Tequisquiapan y El Charcón, lo cual permite el depósito de materia orgánica en el sustrato. Esta condición parece favorecer a T. mucrontum, ya que ambos rodales presentaron una estructura arbórea media o alta (Cuadro 3) con un incremento radial elevado. Por otro lado, ambos sitios mostraron una correlación alta, con respecto a la producción de ramas y producción de conos por individuo (Cuadro 4), lo cual indica que las podas y la contaminación influyen significativamente en su crecimiento y reproducción. Kozlowski y Pallardy (1997) mencionan que son varios los factores medioambientales que influyen en el crecimiento reproductivo de especies leñosas, siendo los principales la intensidad de la luz, disponibilidad del agua, temperatura, fertilidad del suelo, salinidad y contaminación, además de que se ha comprobado que las inundaciones prolongadas del sustrato disminuyen la floración, producción de semillas y calidad del fruto. En este sentido, durante el periodo del 2002 al 2003, las estaciones cercanas a estos rodales, Paso de Tablas y El Salitre, registraron una temperatura promedio de 17 a $21^{\circ} \mathrm{C}$ (Fig. 3 ), dando pauta a considerar que esta variable también influye en su crecimiento radial, producción de estructuras vegetales y reproductivas. Es sabido que la temperatura acelera la descomposición de la materia orgánica por la acción bacteriana, produciendo principalmente fosfatos y nitratos, elementos esenciales en el crecimiento de las plantas; sin embargo, el argumento de que la fijación de nutrientes provenientes de la descarga de drenes permitió un incremento en la biomasa y en su producción de conos, es una conclusión prematura que necesita ser comprobada.

Al parecer, los valores elevados de cobertura advierten una mayor producción de ramas. Esto se logró apreciar en Panales y El Trapiche, cuya producción de ramas principales por individuo fue la más alta $(13.82 \pm 1.95$ y $11.67 \pm$ 0.56 respectivamente), seguidos de Puente de Ayutla ( 8.78 \pm 1.05 ); así como en los árboles considerados para el análisis de brotes, ya que hubo una relación significativa con el número de ramas encontradas por metro cúbico (primera medición $r=0.599, p=0.002$; segunda medición $r=0.433$, $p=0.034)$; situación que se encuentra también a merced de diversos aspectos genéticos, ambientales y a perturbaciones causadas por el hombre. A pesar de que Panales y El Trapiche presentaron valores altos en cuanto a ramas principales, no se encontró una asociación lineal significativa con la producción de conos (Cuadro 4), lo cual puede reflejar las diferencias genéticas propias de cada rodal, o 
bien, que el efecto de la temperatura pudo influir en ello, ya que la estación meteorológica Ayutla tuvo un registro promedio anual mayor en el 2002 y $2003\left(25^{\circ} \mathrm{C}\right)$, seguida por la estación Tolimán con un valor cecano a $22^{\circ} \mathrm{C}$ (Fig. 3). La correlación más baja la presentó Barranca de Amealco/ SJR (bien conservado) en el 2002, y Presa de Galindo 2 en el 2003, lo cual sugiere que el control del flujo del agua no afecta de manera preponderante la producción de ramas por individuo, ni condiciona la producción de conos. En el caso de Presa de Galindo 1 (ligeramente perturbado), el elevado número de árboles jóvenes, pudo ocasionar el bajo conteo de conos durante los 2 años de muestreo.

Las diferencias en la producción de semillas pueden atribuirse a los cambios en el régimen de lluvias durante los 2 años de estudio. Durante septiembre del 2003, el estado de Querétaro presentó las lluvias más abundantes registradas desde hace 30 años, lo que ocasionó inundaciones en diversos municipios de la entidad. Es probable que las inundaciones prolongadas en Presa de Galindo 1 hayan disminuido la producción de semillas por cono. Cabe destacar que en general la producción de semillas de T. mucronatum se ajustó al registro de Carranza (1992) de 20 a 60 semillas en promedio por cono. Los resultados indican que la temperatura, disponibilidad de agua, efecto de las inundaciones y la presencia de materia orgánica en el sustrato afectan el crecimiento vegetativo y por ende el reproductivo; sin embargo, el genotipo puede tener un papel fundamental en este desempeño.

Los cambios hidrológicos-geomórficos son un factor de perturbación sobre la vegetación riparia que puede afectar su crecimiento, ya que el arrastre de materiales finos, piedras y troncos, modifica la estructura del arbolado (Gecy y Wilson, 1990). En México, Treviño et al. (2001) encontraron que en una corriente rápida, con lecho amplio, rocoso y aguas poco profundas, la relación de carga (relación del ancho de copa con el diámetro a 1.30 $\mathrm{m}$ ) en $T$. mucronatum es menor, proporcionando mayor estabilidad, es decir, un tronco robusto en relación con el índice de copa (relación entre el largo y ancho de ésta), y viceversa, en una corriente lenta de aguas profundas. Esta situación se pudo advertir en Presa de Galindo 1, que exhibió valores bajos en cuanto a estructura, si bien es un rodal maduro, y también abundaron los árboles jóvenes. Cuando el embalse se libera produce corrientes rápidas, factor que llega a influenciar el crecimiento de los sabinos. El rodal situado después de la presa mostró un área basal y cobertura mayor, en comparación al ubicado antes del embalse (Cuadro 2). Esta expresión fisonómica se debe quizás a la necesidad de mantener un fuste robusto ante las corrientes producidas cuando se abre la compuerta de la presa.

Llama la atención que Puente de Ayutla, a pesar de que no es un rodal joven, presentó valores muy bajos en cuanto a área basal y cobertura (Cuadro 2); resultados que indican que probablemente los embates del río Concá en temporada de lluvias, han ejercido cambios fisonómicos en los sabinos para mantener una estabilidad de fuste con relación a su copa. Al revisar los datos meteorológicos, se puede distinguir que la estación de Ayutla registró en el periodo de 1984 a 2004 los valores más altos en cuanto a precipitación promedio anual (Fig. 3). Caso semejante Barranca de Amealco/SJR, considerado como el rodal más viejo, distinguiéndose por temporadas de mayor precipitación, donde el espejo de agua se eleva hasta 2 o 3 metros.

De acuerdo con Allan (1995) los sistemas riparios son dinámicos y cambian constantemente tanto en el aspecto ecológico como en el geológico, pero los cambios provocados por el hombre superan en magnitud a los producidos de forma natural. Sin embargo, existen especies riparias como Taxodium distichum, Fraxinus nigra y del género Populus que logran desarrollarse en un amplio rango de condiciones hidrológicas, como áreas permanentemente inundadas, sustratos elevados con un buen drenaje, así como suelos con una rápida sedimentación (Bradley y Smith, 1985; Keeland et al., 1997; Tardif y Bergeron, 1999; Taylor et al., 1999). Las observaciones y los resultados obtenidos en este trabajo, sugieren que T. mucronatum también logra establecerse en un amplio rango de condiciones hidrológicas, geomorfológicas e inducidas por el hombre, dando pauta de hacia dónde deben dirigirse las nuevas líneas de investigación.

\section{Agradecimientos}

A la Fundación Internacional para la Ciencia (International Foundation for Science), por financiar parcialmente este trabajo a través del Proyecto D/2816-2F. Al M. en C. Ulises Padilla García, técnico investigador de la Universidad Autónoma de Querétaro, por la elaboración del mapa temático de ubicación de los sitios de estudio.

\section{Literatura citada}

Allan, J. D. 1995. Stream ecology structure and function of running waters. Kluwer Academic, Dordrecht p. 305-343.

Alpert, P., F. T. Griggs, and D. R. Peterson. 1999. Riparian forest restoration along large rivers: Initial results from the Sacramento River Project. Restoration Ecology 7:360-368.

Bradley, C. E. y D. G. Smith. 1985. Plains cottonwood recruitment and survival on a prairie meandering river floodplain, Milk River, Southern Alberta and Northern Montana. Canadian Journal of Botany 64:1433-1442. 
Brown, S. 1981. A comparison of the structure, primary productivity, and transpiration of cypress ecosystem in Florida. Ecological Monographs 51:403-427.

Carranza, E. 1992. Familia Taxodiaceae. Flora del Bajío y de Regiones Adyacentes. Fascículo 4. Instituto de Ecología, A. C. Centro Regional del Bajío, Pátzcuaro, Michoacán. 7 p.

Comisión Nacional del Agua. 2001. Índice de Calidad de Agua de acuerdo a su Uso. Reporte técnico. Subdirección General Técnica. Gerencia de Saneamiento y Calidad de Agua. Querétaro.

Farjon, A., C. N. Page y N. Schellevis. 1993. A preliminary world list of threatened conifer taxa. Biodiversity and Conservation 2:304-326.

Fritts, H. C. 1976. Tree rings and climate. Academic Press. London, United Kingdom. 567 p.

Gecy, J. L. y M. V. Wilson. 1990. Initial establishment of riparian vegetation after disturbance by debris flows in Oregon. American Midland Naturalist 123:282-291.

Harms, W. R. 1973. Some effects of soil type and water regime on growth of tupelo seedlings. Ecology 54:188-193.

Hesse, I. D., J. W. Day Jr. y T. W. Doyle. 1998. Long-term growth enhancement of baldcypress (Taxodium distichum) from municipal wastewater application. Enviromental Management 22:119-127.

INEGI, 2004a. Instituto Nacional de Estadística, Geografía e Informática. http://mapserver.inegi.gob.mx/geografia/espanol/ estados/qro/clim.cfm; 03.XI.2004.

INEGI, 2004b. Instituto Nacional de Estadística, Geografía e Informática. http://mapserver.inegi.gob.mx/geografia/espanol/ estados/qro/agri.cfm; 03.XI.2004.

INEGI ( Instituto Nacional de Estadística, Geografia e Informática). 2003a. Cuaderno Estadístico Municipal. Arroyo Seco. INEGI, Aguascalientes, Aguascalientes.

INEGI (Instituto Nacional de Estadística, Geografía e Informática). 2003b.. Cuaderno Estadístico Municipal. Tequisquiapan. INEGI Aguascalientes, Aguascalientes. p. 3-11

INEGI (Instituto Nacional de Estadística, Geografía e Informática). 2001a. Anuario estadístico del estado de Querétaro de Arteaga. INEGI, Aguascalientes, Aguascalientes. 480 p.

INEGI Instituto Nacional de Estadística, Geografía e Informática). 2002a.. Cuaderno Estadístico Municipal. Tolimán. INEGI, Aguascalientes, Aguascalientes. $180 \mathrm{p}$.

INEGI (Instituto Nacional de Estadística, Geografía e Informática). 2002b. Cuaderno Estadístico Municipal. San Juan del Río. INEGI, Aguascalientes, Aguascalientes. 204 p.

INEGI (Instituto Nacional de Estadística, Geografía e Informática). 2001b. Cuaderno Estadístico Municipal. Amealco de Bonfil. INEGI, Aguascalientes, Aguascalientes. 172 p.

INEGI.(Instituto Nacional de Estadística, Geografía e Informática). 2001c.. Cuaderno Estadístico Municipal. Cadereyta de Montes. INEGI, Aguascalientes, Aguascalientes. $176 \mathrm{p}$.

Keeland, B. D., W. H. Conner y R. R. Sharitz. 1997. A comparison of wetland tree growth response to hydrologic regime in Louisiana and South Carolina. Forest Ecology and Management 90:237250

Kozlowski, T. T. y S. G. Pallardy. 1997. Growth control in woody plants. Academic, New York. p. 323-351.

Kozlowski, T. T. 1985. Soil aireation, flooding and tree growth. Journal of Arboriculture 11:85-96.

Malanson, G. P. 1993. Riparian landscapes. Cambridge University Press, 296 p.

Martínez, M. 1963. Las pináceas mexicanas, tercera edición. Instituto de Biología, UNAM, México, D.F. 400 p.

Naiman, R. J. y H. Décamps. 1997. The ecology of interfaces: riparian zones. Annual Review of. Ecology and Systematics 28: 621-658.

Nilsson, C. y M. Svedmark. 2002. Basic principles and ecological consequences of changing water regimes: riparian plant communities. Environmental Management 30:468-480.

Olvera, V. M., S. Moreno G., y B. Figueroa R. 1996. Sitios permanentes para la investigación silvícola. Universidad de Guadalajara/Instituto Manantlán de Ecología y Conservación de la Biodiversidad/Departamento de Ecología y Recursos Naturales, Guadalajara, Jalisco. 55 p.

Patten, D. T. 1998. Riparian ecosystems of semi-arid North America: diversity and humans impacts. Wetlands 18:498-512.

Rzedowski, J. 1978. Vegetación de México. Limusa, México, D.F. $432 \mathrm{p}$.

SAGARPA, 2003. Secretaría de Agricultura, Ganadería, Desarrollo Rural, Pesca y Alimentación. www.sagarpa.gob.mx/ dlg/ queretaro/nuestro.htm; última consulta: 01.VI.2004.

SAS Institute Inc., 1995. Statistics and graphics guide JMP. Statistical discovery software. Cary, North Carolina.

Schlesinger, W. H. 1978. Community structure, dynamics and nutrient cycling in the Okefenokee cypress swamp-forest. Ecological Monographs 48: 43-65.

Suzán-Azpiri,H., G. Enríquez- Peña, G. Malda-Barrera. 2007. Population structure of the mexican baldcypress (Taxodium mucronatum ten.) in Queretaro, Mexico. Forest Ecology and Management 242:243-249.

Tardif, J. y Y. Bergeron. 1999. Population dynamics of Fraxinus nigra in response of flood-level variations, in north-western Quebec. Ecological Monographs 69:107-125.

Taylor, J. P., D. B. Wester y L. M. Smith. 1999. Soil disturbance, flood management, and riparian woody plant establishment in the Rio Grande floodplain. Wetlands 19:372-382.

Trenard, Y. 1982. Making wood speak: an introduction to dendrocronology. Foresty Abstracts 43: 729-759.

Treviño G., E. J., C. Cavazos C. y O. A. Aguirre C. 2001. Distribución y estructura de los bosques de galería en dos ríos del centro sur de Nuevo León. Madera y Bosques 7: 3-25.

Zamudio, S., J. Rzedowski, E. Carranza G., y G. Calderón. 1992. La vegetación en el estado de Querétaro. CONCYTEQ/Instituto de Ecología A. C.-Centro Regional del Bajío, Pátzcuaro, Michoacán. Impreso por el Gobierno del Estado de Querétaro. 92 p. 


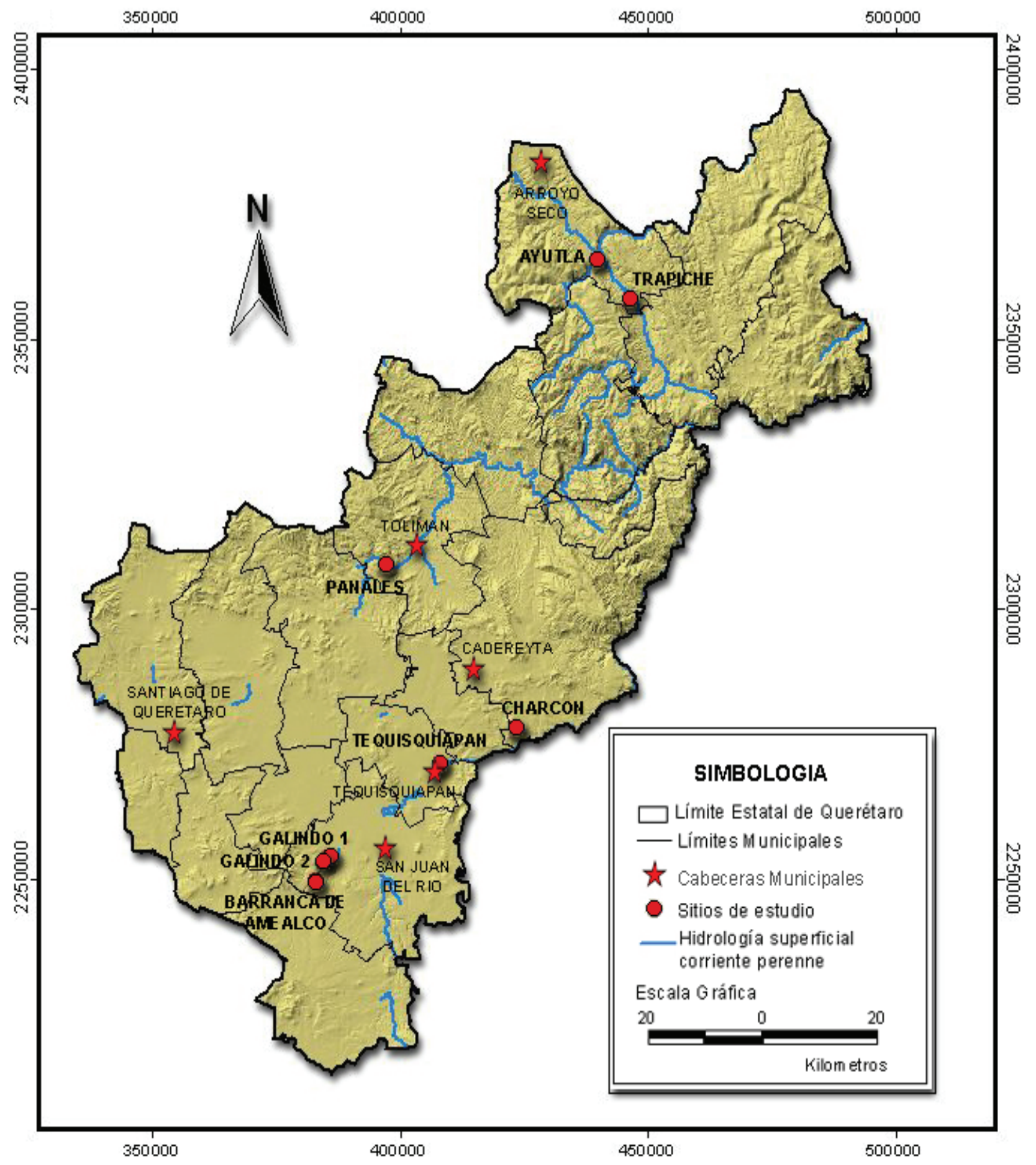

Figura 1. Ubicación de los sitios de muestreo. Corrientes permanentes y semipermanentes en el estado de Querétaro. Comisión Nacional Forestal (CONAFOR). Inventario Nacional Forestal (2000). 1, Panales; 2, Barranca de Amealco/San Juan del Río; 3 , Presa de Galindo 1; 4. Presa de Galindo 2; 5, El Trapiche; 6, Puente de Ayutla; 7, Tequisquiapan; 8, El Charcón. 


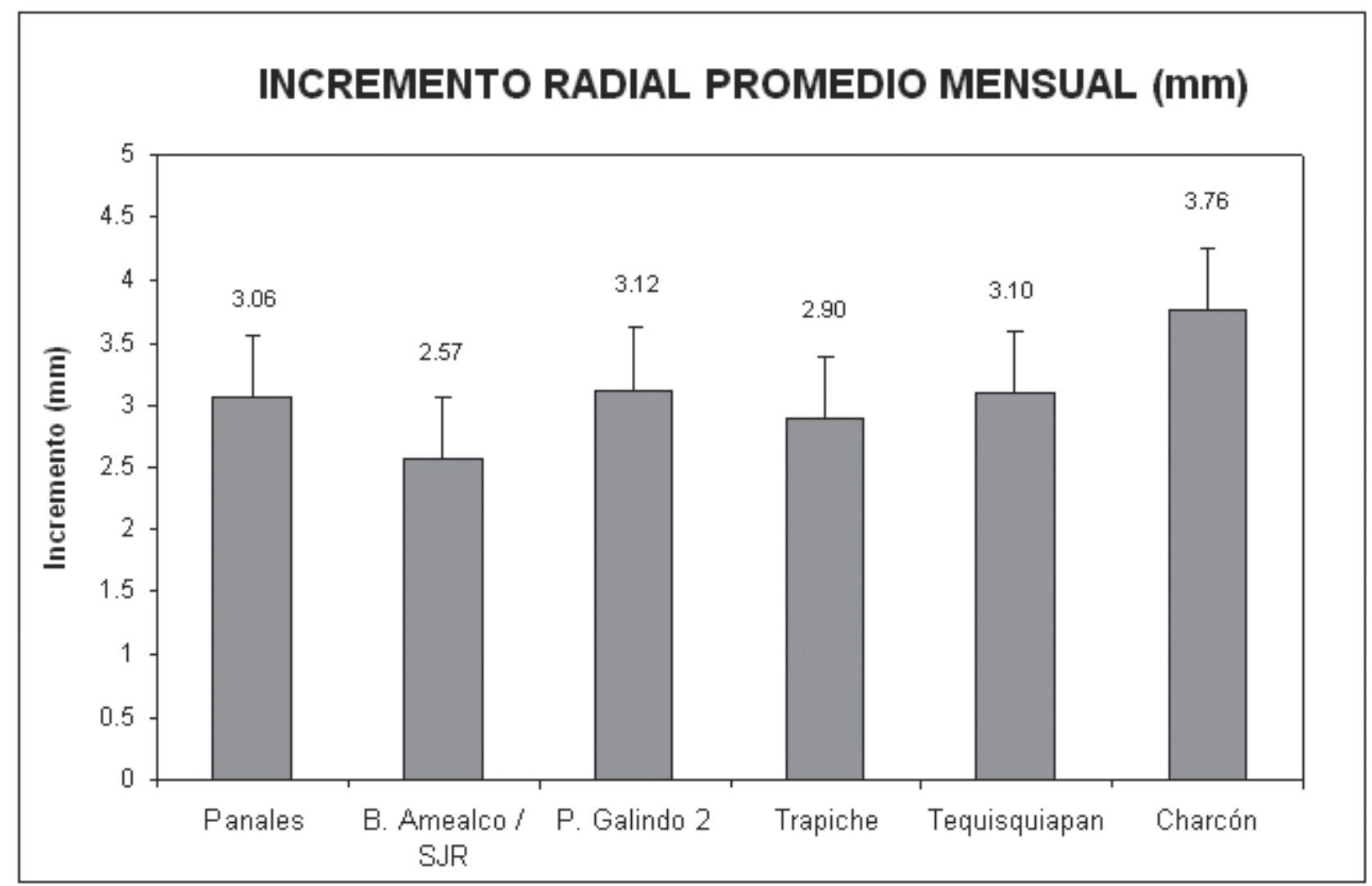

Figura 2. Incremento radial promedio mensual (mm) de Taxodium mucronatum en el estado de Querétaro.

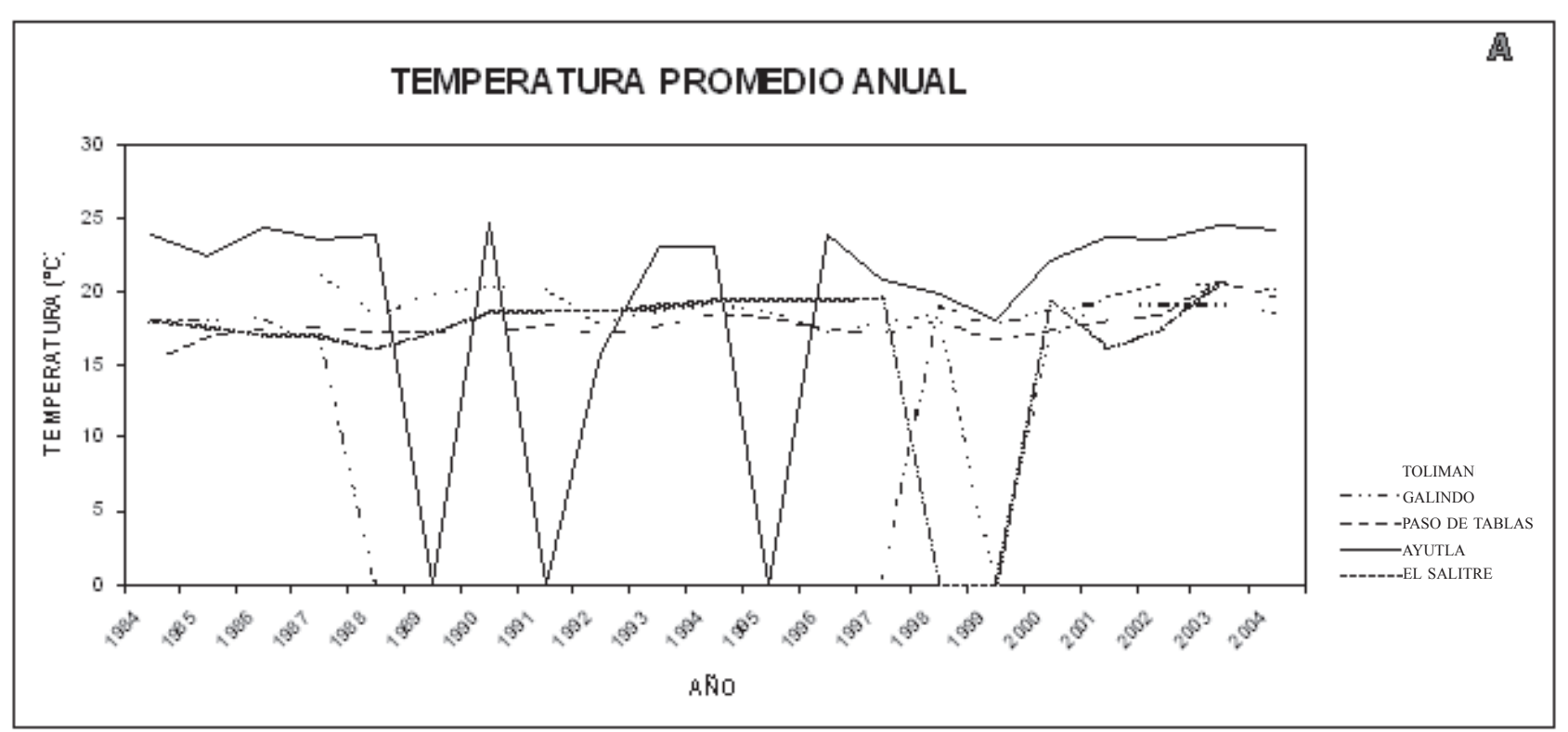

Figura 3. A, temperatura promedio anual. 


\section{PRECIPITA CIÓN PROMEDIO ANUAL}

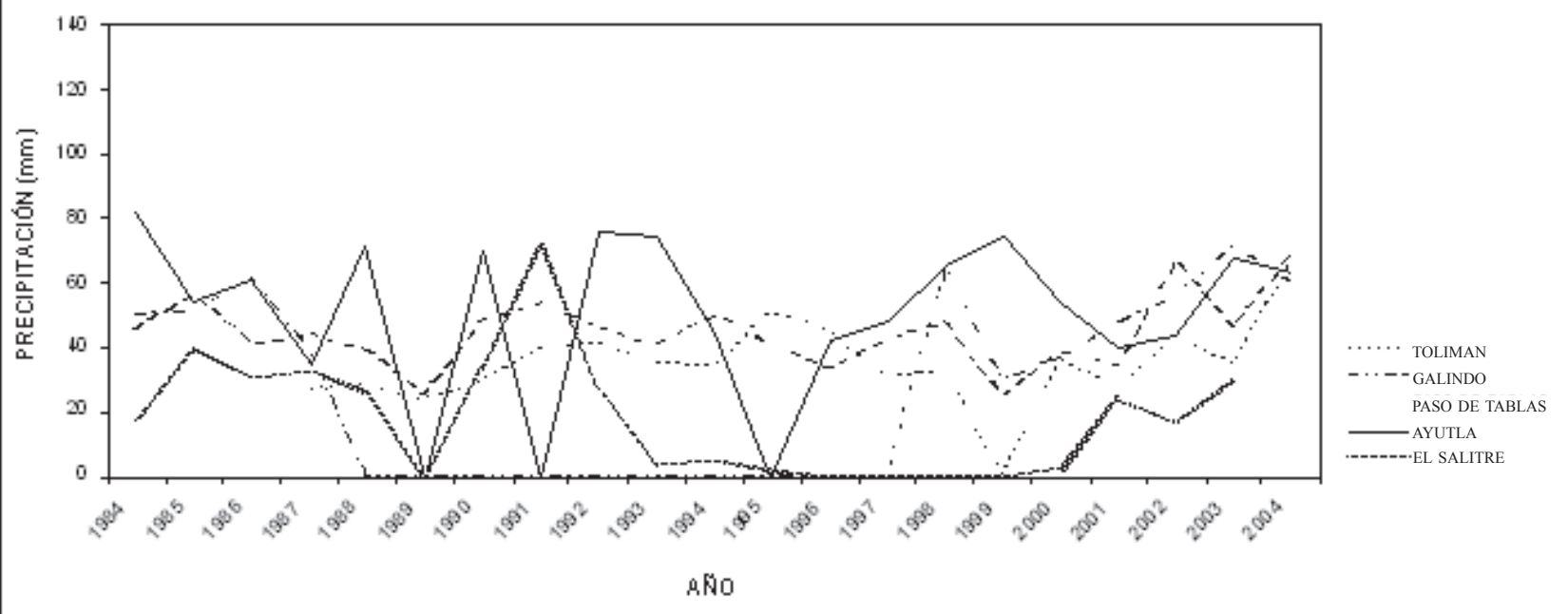

Figura 3. B, precipitación promedio anual durante el periodo comprendido entre los años 1984 a 2004, de 5 estaciones meteorológicas en el estado de Querétaro. Fuente: Comisión Nacional del Agua, Gerencia Estatal en Querétaro, Subgerencia de Ingeniería (Los registros graficados como cero representan ausencia de datos).

\section{Promedio de conos producidos por individuo}

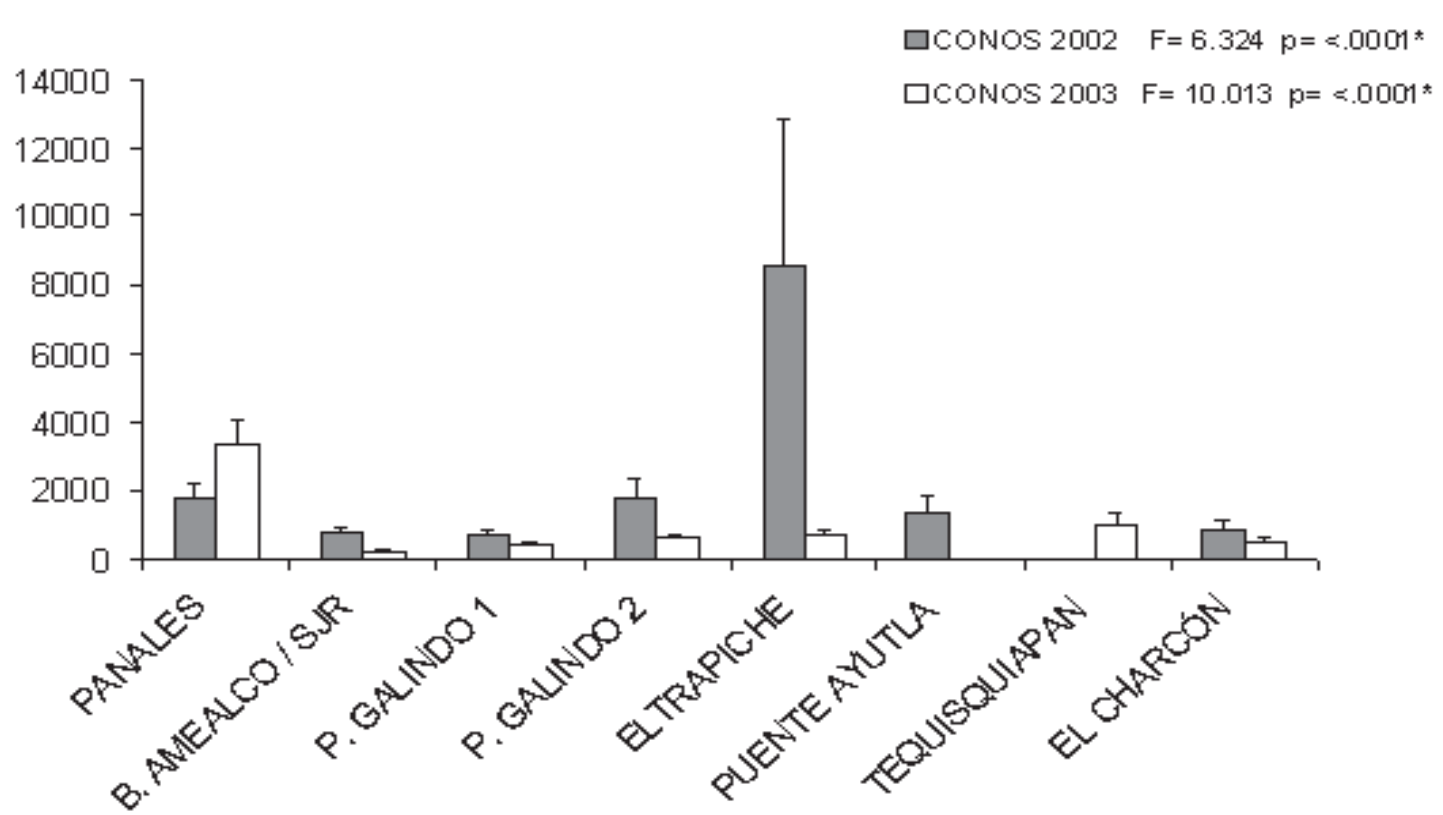

Figura 4. Promedio de conos de Taxodium mucronatum producidos por individuo durante los años 2002 y 2003 , en el estado de Querétaro. 


\section{CREC IMEN TO DE BROTES - PRIMERA MEDICIÓN}

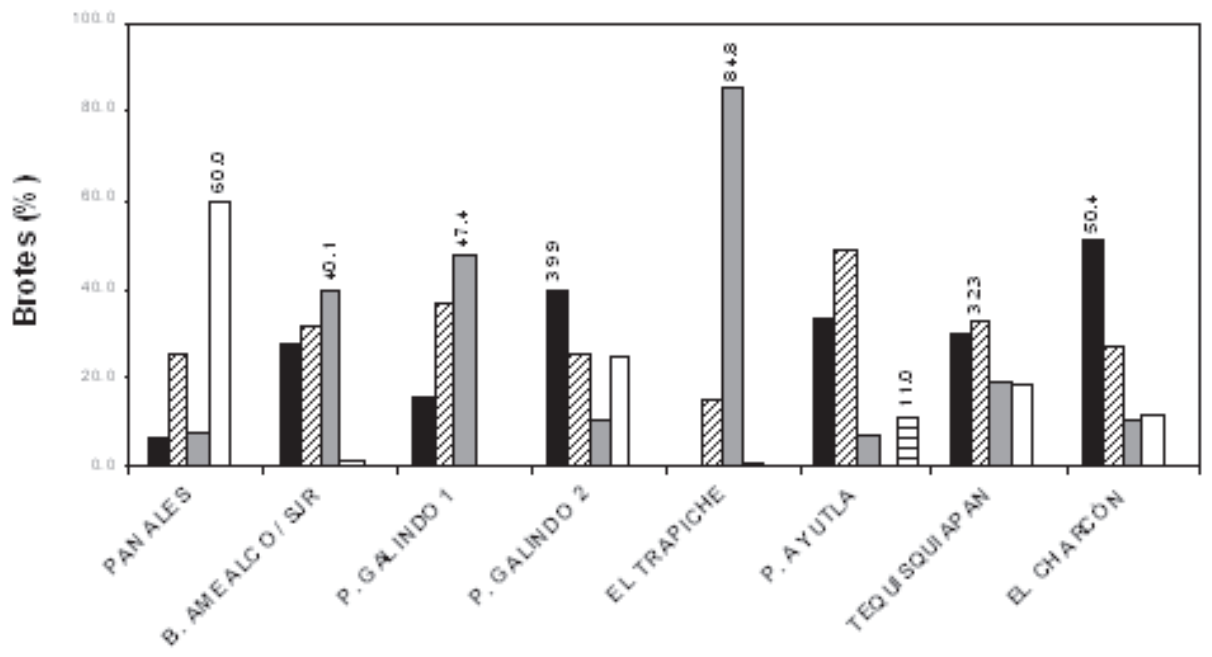

\section{Longttud}

घ1) $20-30 \mathrm{~cm}$

๒2) $31-50 \mathrm{~cm}$

口3) $51-70 \mathrm{~cm}$

प4) $71-100 \mathrm{~cm}$

曰5) $>100 \mathrm{~cm}$

CRE CIMENTO DE BROTES - SEGUND A MEDICIÓN

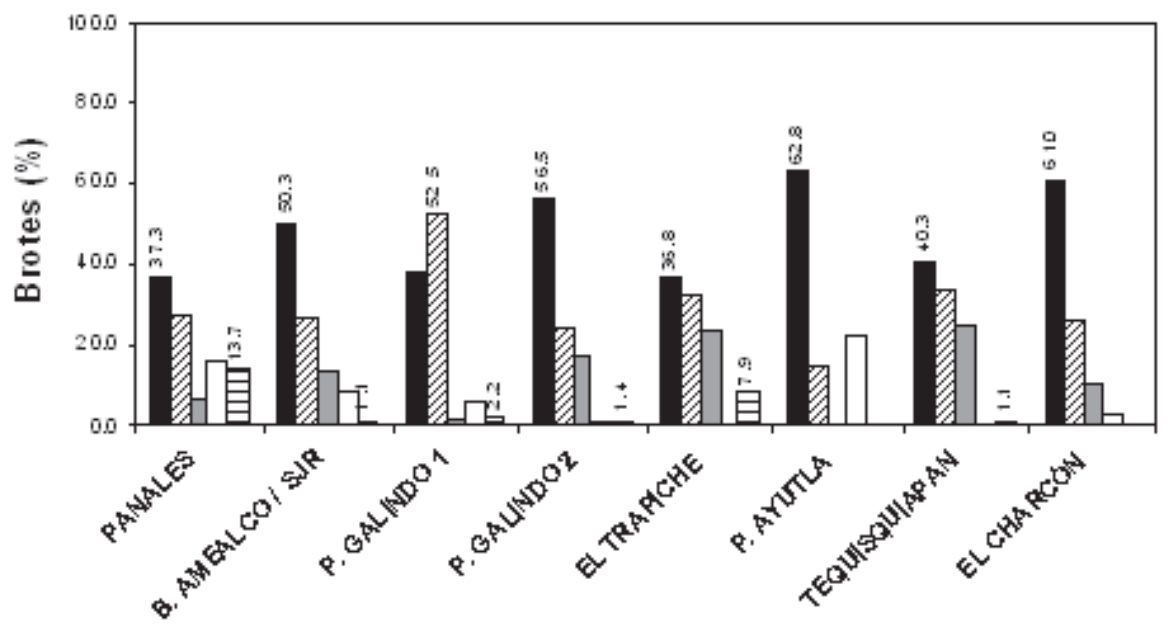

Figura 5. Clasificación del crecimiento de brotes de Taxodium mucronatum en 5 categorías de longitud, en el estado de Querétaro. 
